\title{
Integrating power quality analysis and protection relay functions
}

\author{
A. Lisowiec ${ }^{1}$, A. Nowakowski ${ }^{1}$ and Z. Kołodziejczyk ${ }^{1}$ \\ ${ }^{1}$ Center of Teleinformation Systems and Hardware Applications \\ Tele and Radio Research Institute \\ 11 Ratuszowa, 03-450 Warsaw (Poland) \\ Phone: +48 2261922 41, ext. 286, fax: +48 226197314 \\ E-mails: alisow@itr.org.pl, anowakow@itr.org.pl, zdzich@itr.org.pl
}

\begin{abstract}
In the paper the implementation of power quality analysis functions in protection relay has been presented. The main focus of the research has been on the development of efficient algorithms for voltage and current signals frequency spectrum determination with harmonic and interharmonic content up to $2 \mathrm{kHz}$. The technique that enables efficient implementation of frequency spectrum determination in protection relay employed in systems with varying line frequency is resampling in the digital domain. The accuracy analysis of the resampling technique applied in a protection relay, taking into account implementation issues, has been carried out.
\end{abstract}

\section{Key words}

power quality, protection relay, digital resampling

\section{Introduction}

The amount of energy extracted from renewable sources, e.g. wind or hydro power plants, photovoltaic arrays, fuel cell systems is growing. These sources must be either synchronized or converted to alternating current before their energy can be injected into the grid. During this process disturbances in the form of harmonics, voltage sags and overshoots are created which have to be kept under control. There will be a growing need for power quality metering devices placed at nodes where local power sources connect to the grid. As the power quality measurement process is a demanding one, power quality analyzers have developed into specialized, complex devices. Power quality analyzers available on the market today are too expensive and specialized to be applied on a mass scale.

The objectives of the work which we try to describe have been to equip a conventional protection relay with power quality analysis functions at a very small additional cost to the end customer. The tremendous advance in the DSP microprocessor technology enables us to use in real time advanced signal processing algorithms like signal resampling in the digital domain that are the key to achieving design objectives. To keep the cost of the developed protection relay low, the developed algorithms should put minimal requirements on hardware.

\section{Power quality analyser architecture}

The architecture of power quality analyzer is shown in Fig. 1. Each voltage input signal is separated into low frequency component with spectrum cut above some frequency $f_{\mathrm{g}}$ (typically $f_{\mathrm{g}}$ is equal to $3 \mathrm{kHz}$ ) and high frequency component with spectrum cut below $f_{\mathrm{g}}$.

The high frequency component is used for detecting and recording transient voltage surges. This is done in a module composed of a high speed A/D converter - with sampling rate above $1 \mathrm{Ms}$ per second per channel, and a FIFO memory. The recording process is triggered when the voltage crosses a threshold value. As transient voltage surges last less than $10 \mathrm{~ms}$, only one line period is recorded. The transient voltage surges recording module is largely independent of the rest of power quality analyzer; in Fig. 1 it consists of blocks located outside the dotted line.

The low frequency component of input current or voltage signal is used for determination of signal characteristics like frequency, RMS value, harmonic content, THD etc.

The power quality analyzer module that processes low frequency component determines basically the same signal parameters as conventional protection relay. The main difference in architecture between the two is that a typical protection relay contains two-state inputs module and output relays module while power quality analyzer has much more storage memory.

While the architectures of power quality analyzer and protection relay are very similar (with the exception of transient voltage surge module), both devices differ very much in software. Protection relay contains protection algorithms software while power quality analyzer contains statistical software. Power quality analyzer also has wider input bandwidth to measure harmonic and interharmonic content of the signal up to $2 \mathrm{kHz}$.

Protection relay with $2 \mathrm{kHz}$ bandwidth input signal path, equipped with advanced digital processing and statistical software can replace power quality analyzer. Extremely fast DSP processor and high density memory chips enable concurrent performance of protection and quality analysis functions. 


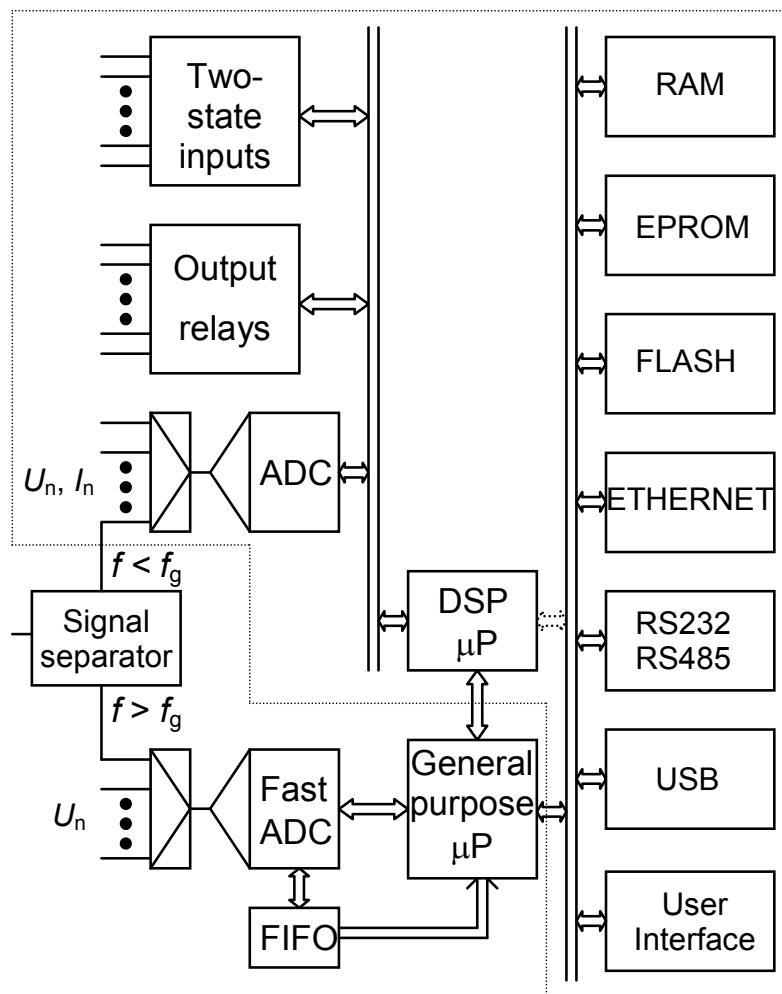

Fig. 1 Block diagram of power quality analyzer

\section{Spectrum determination algorithms for power quality analysis}

\section{A. Sampling frequency choice}

The international standards concerning power quality analysis [1],[2],[3] define precisely which parameters of line voltage and current are to be measured and the preferred methods of measurement in order to determine power quality. In compliance with these requirements, for harmonic content determination, power quality analyzers employ sampling procedures with sampling frequency precisely synchronized to the exact multiple of line frequency. This is necessary for correct spectrum determination as it is known from Fourier theory [4]. If the sampling frequency is not equal to the exact multiple of line frequency, the spectral components are computed with error and false components appear in the spectrum. For interharmonic content determination in $50 \mathrm{~Hz}$ systems the measurement interval should equal 10 line periods which gives $5 \mathrm{~Hz}$ spectrum resolution.

Efficient computation of signal spectrum with the use of FFT transform demands that the number of samples in the measurement interval should equal the power of two. This is however impossible to satisfy both in one line period measurement interval and ten line periods measurement interval. Some power quality analyzers available on the market offer interharmonic content measurement over 8 or 16 line periods interval.

Another disadvantage of synchronizing sampling frequency to signal frequency is the inability to record the signal waveform and preserve the time scale.

A better method to reconcile the requirements on sampling frequency caused by varying line frequency and measurement intervals of different length is to use constant sampling frequency and employ digital multirate signal processing techniques.

The sampling frequency should be chosen with the aim of simplifying the antialiasing filters that precede the analog to digital converter. The bandwidth of the signal that has to be accurately reproduced is $2 \mathrm{kHz}$, so the cutoff frequency of the antialiasing filter should be considerably higher than that. On the other hand the signal frequency components at half the sampling frequency should be adequately suppressed. Assuming that simple two pole $\mathrm{RC}$ antialiasing filter is to be used the sampling frequency should equal at least $16 \mathrm{kHz}$.

\section{B. Digital multirate signal processing in protection relay}

In multirate digital signal processing [4] it is possible to change the sampling rate by a rational factor $N / M$ using entirely digital methods. The input signal sampled with constant frequency $f_{\mathrm{s}}$ is first interpolated by a factor of $N$ and then decimated by a factor of $M$ - both these processes are called collectively resampling. The output sequence consists of samples representing input signal sampled at an effective frequency $f_{\text {seff }}$, where

$$
f_{\text {seff }}=f_{\mathrm{s}} \cdot(N / M)
$$

As both the interpolation and decimation expand the Fourier bandwidth of the signal a special low pass filter has to be used to avoid aliasing. This filter is designed as a FIR filter. The number of filter coefficients is generally proportional to the greater of the two values $N$ and $M$ provided they have no common factors. The output samples are obtained by convolving the input samples (with $N-1$ null samples inserted between each consecutive two samples) with impulse response of the filter [4].

The first thing that has to be determined is how many samples are needed to calculate the spectral components of the signal. For protection purposes, the knowledge of harmonics up to $11^{\text {th }}$ is enough. Thus, taking into account that the number of samples, for computational reasons, must equal the power of two, 32 samples per period are enough [4], which means that $f_{\text {seff }}=1600 \mathrm{~Hz}$. For power quality analysis, the EN 61000-4-7 standard demands that the measurement interval should equal ten line periods and the harmonics up to $40^{\text {th }}$ (equivalently interharmonics up to $400^{\text {th }}$ ) have to be calculated. The lowest power of two greater than 800 is 1024 .

At 1024 samples per 10 line periods, the ideal sampling frequency $f_{\mathrm{si}}$ is calculated from the equation

$$
f_{\mathrm{si}}=(f / 10) \cdot 1024=f \cdot 102.4
$$

where $f$ is the line frequency.

The relative accuracy $\operatorname{Er}(f)$, as a function of $f$, with which $f_{\text {seff }}$ approximates $f_{\text {si }}$ is equal to

$$
\operatorname{Er}(f)=1-\left(f_{\mathrm{s}} / f\right) \cdot(1 / 102.4) \cdot(N / M)
$$

The accuracy with which $f_{\text {seff }}$ is to approximate $f_{\mathrm{si}}$ could be determined from simulating how different values of $N$ and $M$ affect the accuracy of spectrum 
determination. However some clues about the values of $N$ and $M$ can be obtained from EN 61000-4-7 standard. In chapter 4.4.1 it states that the time interval between the rising edge of the first sample in a measurement interval (200 $\mathrm{ms}$ in $50 \mathrm{~Hz}$ systems) and the rising edge of the first sample in the next measurement interval should equal 10 line periods with relative accuracy not worse than $0.03 \%$.

With $\operatorname{Er}(f)$ constant and one of the $N$ or $M$ value chosen, the other can be determined from (3) for each value $f$ of interest. The largest values of $N$ and $M$ determine the computational effort of the resampling procedure. Table I contains minimal values of $N$ for which the approximation accuracy determined from (3) is equal to $0.03 \%$ when $f$ ranges from $45 \mathrm{~Hz}$ to $55 \mathrm{~Hz}$, for various values of sampling frequency

TABLE I $N$ as a function of $f_{\mathrm{s}}$

\begin{tabular}{|c|c|c|c|c|c|c|}
\hline$f_{\mathrm{s}}[\mathrm{Hz}]$ & 8000 & 10000 & 12000 & 14000 & 16000 & 20000 \\
\hline$N$ & 1200 & 900 & 800 & 750 & 600 & 500 \\
\hline
\end{tabular}

\section{Spectrum determination accuracy analysis}

The analysis of spectrum determination accuracy has been carried out assuming $f_{\mathrm{s}}=16 \mathrm{kHz}$ and $N=600$. For each of the line frequency $f$ value from $<45 \mathrm{~Hz}, 55 \mathrm{~Hz}>$ interval, with $0.01 \mathrm{~Hz}$ step, the value for $M$ was chosen so as to minimize $\operatorname{Er}(f)$. The minimal values of $\operatorname{Er}(f)$ are presented in Fig. 2.

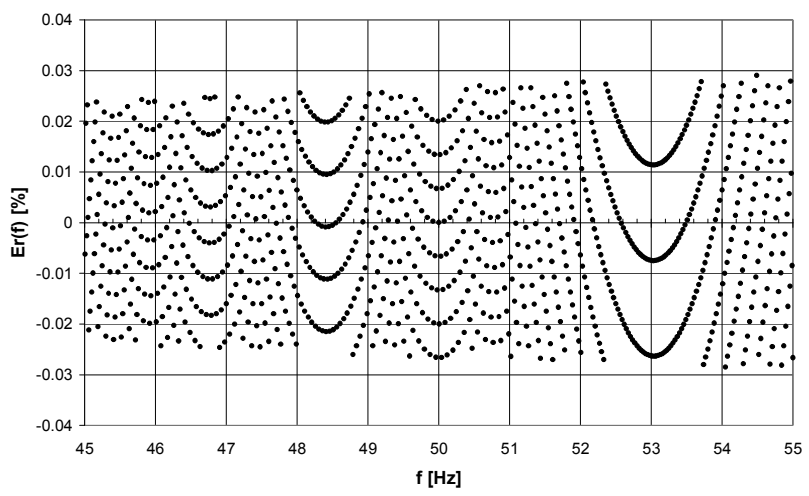

Fig. 2 Error of the effective sampling frequency $f_{\text {seff }}$ relative to ideal sampling frequency $f_{\mathrm{si}}$

The FIR filter was designed using Keiser window [4]. The number of filter coefficients was equal to 37 000. In subsequent calculations the filter coefficients were quantized to 16 bits. Filters of that length are possible to implement in real time in protection relays equipped with modern DSP chips. It means that the computation time of the filter times the number of voltage and current channels (this number varies between 6 and 20 depending on number of protection functions implemented in the relay) takes less than $10 \%$ of processor time.

A large number of simulations were carried out. The simulations were performed with software generated data on fixed point DSP processor implemented in a protection relay. The simulation results for two line frequency values are presented below.

With $f$ equal to $50 \mathrm{~Hz}$ and $N=600, M=1875$ and $\operatorname{Er}(50 \mathrm{~Hz})=0$. In this case the effective sampling frequency $f_{\text {seff }}(=5120 \mathrm{~Hz})$ is equal to the ideal sampling frequency $f_{\text {si }}$.

With $f=54.49 \mathrm{~Hz}$ and $N=600, M=1720$ and $\operatorname{Er}(54.49 \mathrm{~Hz})=0.029 \%$.

Two signal waveforms were subjected to the resampling procedure, waveform I and waveform II.

waveform I

fundamental $-50 \%$ of maximum range

$399^{\text {th }}$ interharmonic $(1995 \mathrm{~Hz}$ for $f=50 \mathrm{~Hz})-$

$10 \%$ relative to fundamental

$40^{\text {th }}$ harmonic $-0.5 \%$ relative to fundamental

$401^{\text {st }}$ interharmonic $-0.2 \%$ relative to

fundamental

waveform II

fundamental $-50 \%$ of maximum range

$40^{\text {th }}$ harmonic $(2 \mathrm{kHz}$ for $f=50 \mathrm{~Hz})-0.5 \%$

relative to fundamental

\section{1) Case $f=50 \mathrm{~Hz}$}

Fig. 3 presents the spectrum of waveform I quantized to 12 bits after it has been subjected to the resampling procedure with $f_{\text {seff }}$ equal to $5120 \mathrm{~Hz}$.

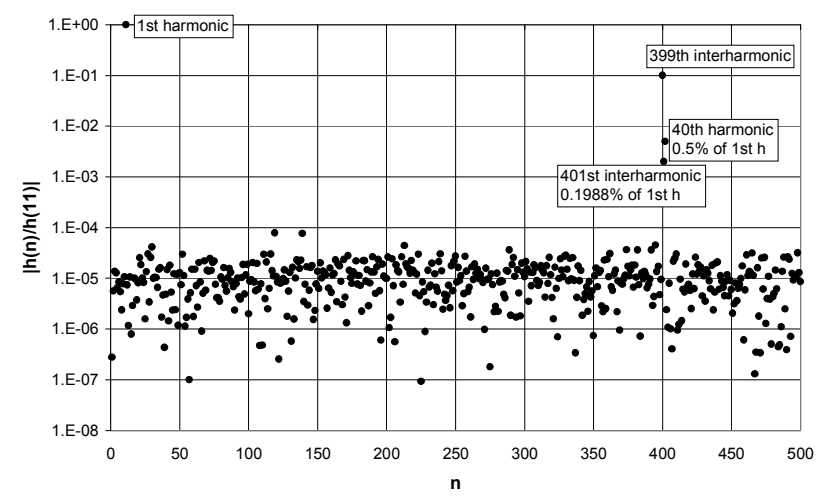

Fig. 3 FFT spectrum of waveform $I$ with fundamental equal to $50 \mathrm{~Hz}$, quantized to $12 \mathrm{bits}$, after resampling with $N=600$ and $M=1875$

As can be seen from Fig. 3, the accuracy of spectrum determination after resampling is almost perfect and more than sufficient for power quality analysis [2]. The error in $401^{\text {st }}$ interharmonic is $1 \%$. The spectrum noise floor at $80 \mathrm{~dB}$ relative to the fundamental is due mainly to the waveform I quantization at 12 bits. In Fig. 4, the spectrum of waveform I quantized to 16 bits and subjected to the same resampling procedure is presented.

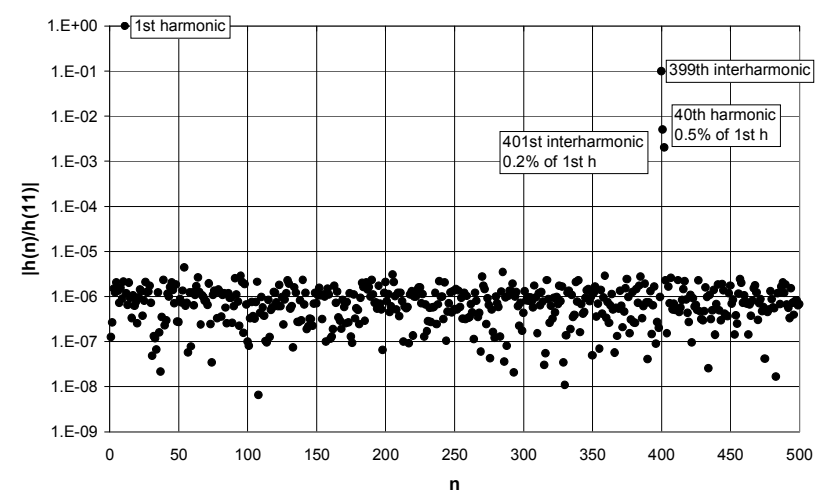

Fig. 4 FFT spectrum of waveform I with fundamental equal to $50 \mathrm{~Hz}$, quantized to 16 bits, after resampling with $N=600$ and $M=1875$ 
At 16 bit waveform quantization the spectrum noise floor is more than $100 \mathrm{~dB}$ below fundamental.

\section{2) Case $f=54.49 \mathrm{~Hz}$}

The ideal sampling frequency for this line frequency according to (2) is equal to $5579.776 \mathrm{~Hz}$. The best approximation of this frequency with $f_{\mathrm{s}}=16000 \mathrm{~Hz}$ and $N=600$ can be achieved with $M=1720$ with relative error $\operatorname{Er}(54.49 \mathrm{~Hz})$ equal to $0.029 \%$. The Fourier theory predicts that the spectrum computed after resampling procedure contains errors [4] and the simulation results that follow show that this is indeed the case.

Fig. 5 presents the spectrum of waveform $I$, quantized to 12 bits, after it has been subjected to the resampling procedure with $f_{\text {seff }}$ equal to $5581.395349 \mathrm{~Hz}$.

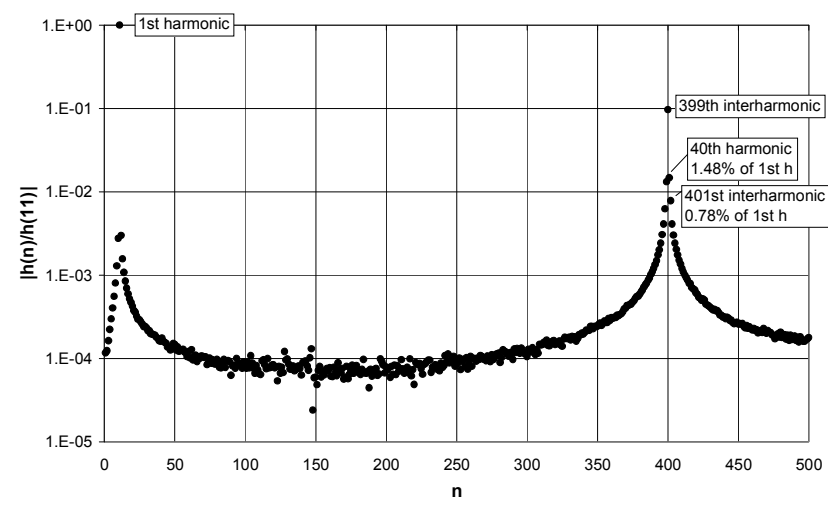

Fig. 5 FFT spectrum of waveform $I$ with fundamental equal to $54.49 \mathrm{~Hz}$, quantized to 12 bits, after resampling with $N=600$ and $M=1720$

The level of $399^{\text {th }}$ interharmonic is very close to the true value. However the level of $40^{\text {th }}$ harmonic is almost three times higher than the true value and the level of $401^{\text {st }}$ interharmonic is almost four times higher than the true value. The observed effect can be explained by leakage of the spectrum from $399^{\text {th }}$ interharmonic of relatively large level to neighbouring interharmonics [4].

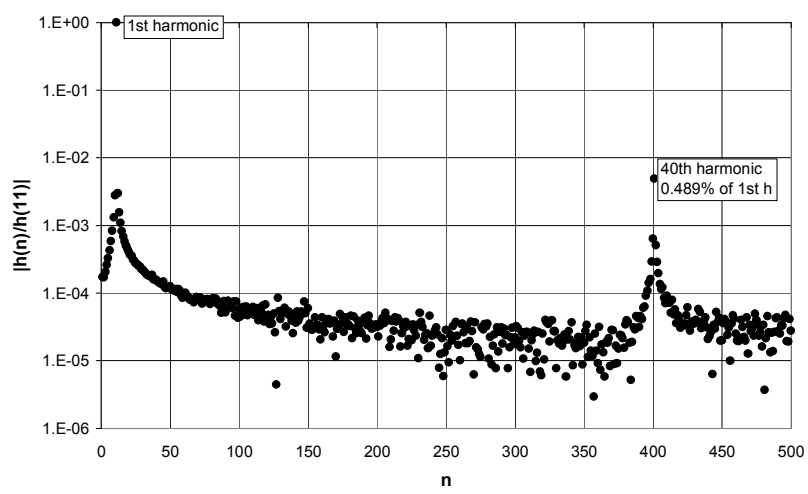

Fig. 6 FFT spectrum of waveform $I$ with fundamental equal to $54.49 \mathrm{~Hz}$, quantized to 12 bits, after resampling with $N=600$ and $M=1720$

Fig. 6 shows the spectrum of waveform II, quantized to 12 bits, after resampling, with $f_{\text {seff }}=5581.395349 \mathrm{~Hz}$. In this case only $40^{\text {th }}$ harmonic together with fundamental were present in the original signal. The level of $40^{\text {th }}$ harmonic after resampling, calculated by FFT, is equal to the true value with $2.4 \%$ error.
To determine whether the error in spectrum determination was caused by the resampling procedure, the waveforms $I$ and $I I$ were sampled at sampling frequency $f_{\mathrm{s}}$ equal to $5581.395349 \mathrm{~Hz}$. The resulting sampled sequences, quantized to 12 bits, were subjected to FFT transform and the resulting spectrums are presented in Figures 7 and 8.

The comparison of Figures 5 and 7 shows that the same inaccuracies in spectrum determination occur regardless of the method in which samples of the waveform were obtained as long as the sampling frequencies were equal. Comparison of Figures 6 and 8 shows that in the absence of interharmonics both methods, e.g. resampling in digital domain and synchronization of sampling frequency to multiple of line frequency give correct results in spectrum determination.

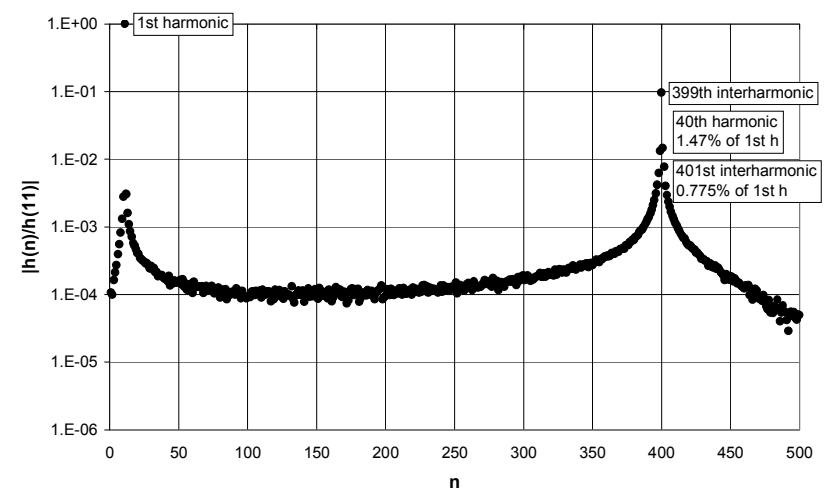

Fig. 7 FFT spectrum of waveform $I$ with fundamental equal to $54.49 \mathrm{~Hz}$, quantized to $12 \mathrm{bits}$, after sampling with frequency equal to $5581.395349 \mathrm{~Hz}$

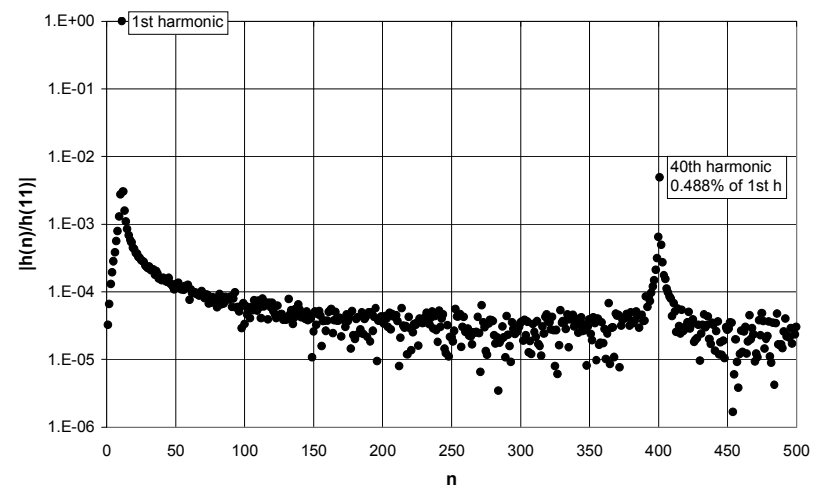

Fig. 8 FFT spectrum of waveform II with fundamental equal to $54.49 \mathrm{~Hz}$, quantized to $12 \mathrm{bits}$, after sampling with frequency equal to $5581.395349 \mathrm{~Hz}$

\section{Remarks}

Accuracy analysis carried out showed, that for spectrum determination with FFT technique, constant sampling frequency with digital resampling is equivalent to synchronization of sampling frequency to multiple of line frequency. If the effective sampling frequency $f_{\text {seff }}$ after resampling is equal to synchronized sampling frequency $f_{\text {synchro, }}$, the discrete spectrums from two sample sequences are essentially identical.

The accuracy of spectrum determination, particularly with high interharmonic content depends on the accuracy with which effective sampling frequency $f_{\text {seff }}$ 
approximates ideal sampling frequency, which is equal to the multiple of the reciprocal of line frequency times the number of samples (2).

The EN 61000-4-7 standard requires that the effective sampling frequency $f_{\text {seff }}$ should approximate the ideal sampling frequency with the relative error less than $0.03 \%$. However, as was shown above, such error in ideal frequency approximation is too large when accurate determination of closely spaced interharmonic components in signal spectrum is needed.

\section{Averaging interval versus line frequency}

Standards concerning power quality analysis [2], [3] specify averaging algorithms of voltage RMS values. The RMS value should be computed over one line period interval every half line period. The start of every measurement interval should be synchronized to the rising or falling edge of voltage waveform like it is shown in Fig. 10 a). Such method of measurement is easy to implement when the sampling frequency is synchronized to the multiple of line frequency.

In systems with multirate digital signal processing which employ resampling techniques, the sequence of samples with constant number of samples per period is not available. Thus it is impossible to synchronize the RMS measurement interval with line frequency. The start of each RMS measurement interval (grey rectangle in Fig. 10) occurs every $10 \mathrm{~ms}$ determined by a precision clock (vertical lines in Fig. 10). Depending on the line frequency, the RMS measurement interval is slightly longer (line frequency less than $50 \mathrm{~Hz}$, Fig. $10 \mathrm{c}$ )) or slightly shorter (line frequency greater than $50 \mathrm{~Hz}$, Fig. $10 \mathrm{~b})$ than $20 \mathrm{~ms}$.

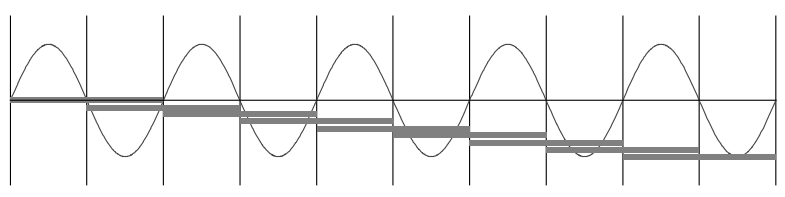

a)

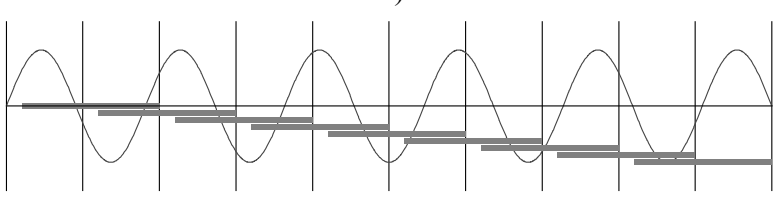

b)

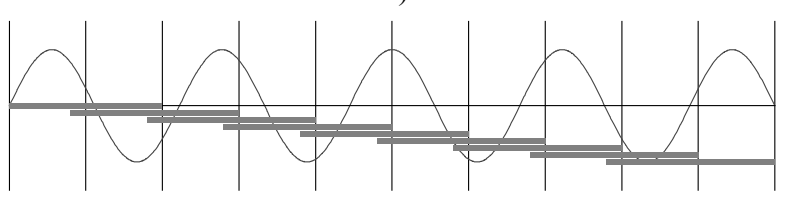

c)

Fig. 10 Overlapping of RMS measurement intervals versus line frequency $f$ : a) $f=50 \mathrm{~Hz}$, b) $f=55 \mathrm{~Hz}$, c) $f=45 \mathrm{~Hz}$
Apparently this is not quite in accordance with relevant standards. Further research into how these effect influences the statistics of power quality parameters is needed.

\section{Conclusion}

Power quality analysis functions can be divided into three categories. One category is transient voltage surges detection and registration. The second category consists of functions that determine how the RMS value of voltage signal behaves over time. The functions in the third category deal with frequency spectrum determination and they are the most demanding in terms of the processing power required.

The research has shown that it is possible to implement functions that compute in real time frequency spectrum of voltage and current signals with harmonic and interharmonic content up to $2 \mathrm{kHz}$ in a conventional protection relay. The algorithms that have been developed for that purpose use advanced signal processing techniques like resampling carried out entirely in the digital domain.

The main advantage of developed algorithms is that they put minimal requirements on hardware. Only one constant sampling frequency is needed both for protection functions and power quality analysis functions.

\section{Acknowledgement}

This research work has been financed by public money for science in the years $2007-2008$ as a targeted research project.

\section{References}

[1] European Standard EN 50160: Voltage characteristics of electricity supplied by public distribution systems

[2] European Standard EN 61000-4-7:2002: Electromagnetic compatibility (EMC) Part 4-7: Testing and measurement techniques - General guide on harmonics and interharmonics measurements and instrumentation, for power supply systems and equipment connected thereto

[3] European Standard EN 61000-4-30:2003: Electromagnetic compatibility (EMC) Part 4-30: Testing and measurement techniques - Power quality measurement methods

[4] Oppenheim A.V., Schafer R.W. Discrete-Time Signal Processing, 2ed., PH, 1998 